\title{
Bergman-Hartogs 型域的全纯自同构群
}

\author{
潘利双*, 王安 \\ 首都师范大学数学科学学院, 北京 100048 \\ E-mail: plshuang123@163.com, wangan@cnu.edu.cn \\ 收稿日期: 2014-01-17；接受日期: 2014-11-20; * 通信作者 \\ 国家自然科学基金 (批准号: 11371257) 和北京市自然科学基金 (批准号: 1122010) 资助项目
}

摘要 我们考虑一类以有界对称域 $D$ 为底的 Bergman-Hartogs 型域

$$
\Omega=\left\{\left(w_{(1)}, \ldots, w_{(r)}, z\right) \in \mathbb{C}^{m_{1}} \times \cdots \times \mathbb{C}^{m_{r}} \times D:\left\|w_{(1)}\right\|^{2 p_{1}}+\cdots+\left\|w_{(r)}\right\|^{2 p_{r}}<K_{D}(z, z)^{-q}\right\},
$$

其中 $K_{D}(z, z)$ 是 $D$ 上的 Bergman 核函数, $r \geqslant 1$ 且为正整数, 参数 $p_{1}, \ldots, p_{r}>1$ 和 $q>0$ 为实数. 我 们给出它的全纯自同构群，并且证明当 $r=1$ 时此自同构群为最大全纯自同构群; 当 $r>1$ 时, 若 $\Omega$ 的 全纯自同构变换 $F$ 将 $(0, z) \in\{0\} \times D$ 映到 $\left(0, z^{*}\right) \in\{0\} \times D$, 则 $F$ 在我们给出的全纯自同构群中.

关键词 Bergman-Hartogs 型域 全纯自同构群 有界对称域

MSC (2010) 主题分类 $32 \mathrm{~A} 07,32 \mathrm{M} 05$

\section{1 引言}

设 $\Omega$ 是 $\mathbb{C}^{n}$ 中的有界域, 并记 $\operatorname{Aut}(\Omega)$ 为将 $\Omega$ 映到 $\Omega$ 的双全纯映射的全体构成的集合. 显然, $\operatorname{Aut}(\Omega)$ 中的变换在映射的复合运算下构成一个群, $\operatorname{Aut}(\Omega)$ 称为 $\Omega$ 的全纯自同构群.

域的全纯自同构群是多复变中研究许多问题的有力工具. 例如, 对于函数空间理论, Bergman 核函 数以及域的全纯等价等问题的研究, 全纯自同构群都起到了很大的作用. 因为全纯自同构群的重要性, 所以它被广泛地关注和研究. $\mathbb{C}^{n}$ 中的单位球和单位多圆盘的全纯自同构群是我们所熟知的. 但是确 定一个域的全纯自同构群是很困难的, 尤其是给出最大全纯自同构群. 华罗庚 ${ }^{[1]}$ 给出了 4 类 Cartan 域的全纯自同构群. $\mathrm{Xu}{ }^{[2,3]}$ 给出了两类例外 Cartan 域的全纯自同构群. Ahn 等人 ${ }^{[4]}$ 证明了以 4 类 Cartan 域为底的 Hartogs 域 $\Omega=\left\{(w, z) \in \mathbb{C}^{N} \times D:\|w\|^{2 \mu}<N_{D}(z, z)\right\}$ 的最大全纯自同构群. 由于确 定域的最大自同构群的困难性, 有时人们去构造域的全纯自同构子群. 殷慰萍等人 [5] 给出了华罗庚域 的全纯自同构群的一个子群. Xu 等人 [6] 给出正规 Siegel 域在固定点处的最大连通迷向子群. Liu 和 Tang 给出了 Egg 域 $B_{p, q}=\left\{(z, \ldots, \zeta, w, \xi, \ldots, \eta) \in \mathbb{C}^{m_{1}} \times \cdots \times \mathbb{C}^{m_{s}} \times \mathbb{C}^{k} \times \mathbb{C}^{n_{1}} \times \cdots \times \mathbb{C}^{n_{t}}:\left|z_{1}\right|^{p_{1}}+\cdots+\right.$ $\left.\left|z_{m_{1}}\right|^{p_{1}}+\cdots+\left|\zeta_{1}\right|^{p_{s}}+\cdots+\left|\zeta_{m_{s}}\right|^{p_{s}}+|w|^{2}+\cdots+\left|w_{k}\right|^{2}+\left|\xi_{1}\right|^{q_{1}}+\cdots+\left|\xi_{n_{1}}\right|^{q_{1}}+\cdots+\left|\eta_{1}\right|^{q_{t}}+\cdots+\left|\eta_{n_{t}}\right|^{q_{t}}<1\right\}$, $0<p_{1}<\cdots<p_{s}<2<q_{1}<\cdots<q_{t}<\infty$ 在原点处的最大迷向子群.

我们考虑更一般的情形, 研究一类以有界对称域为底的 Bergman-Hartogs 型域, 给出它的全纯自 同构群, 并证明给出的全纯自同构群是最大自同构群. 首先, 我们给出以有界对称域为底的 BergmanHartogs 型域的定义. 
设 $D$ 是 $\mathbb{C}^{n}$ 中的有界对称域, $K_{D}(z, z)$ 是 $D$ 上的 Bergman 核函数. 定义 $\mathbb{C}^{m+n}$ 中 BergmanHartogs 型域为

$$
\Omega=\left\{\left(w_{(1)}, \ldots, w_{(r)}, z\right) \in \mathbb{C}^{m_{1}} \times \cdots \times \mathbb{C}^{m_{r}} \times D:\left\|w_{(1)}\right\|^{2 p_{1}}+\cdots+\left\|w_{(r)}\right\|^{2 p_{r}}<K_{D}(z, z)^{-q}\right\},
$$

其中, $m=m_{1}+\cdots+m_{r}, r \geqslant 1$ 为正整数, 参数 $p_{1}, \ldots, p_{r}>1$ 和 $q>0$ 为实数. 若记 $w=\left(w_{(1)}, \ldots, w_{(r)}\right)$, $w_{(j)}=\left(w_{j 1}, \ldots, w_{j m_{j}}\right), j=1, \ldots, r, z=\left(z_{1}, \ldots, z_{n}\right)$, 则 $\Omega$ 中的点为

$$
(w, z)=\left(w_{(1)}, w_{(2)}, \ldots, w_{(r)}, z\right)=\left(w_{11}, \ldots, w_{1 m_{1}}, w_{21} \ldots, w_{2 m_{2}} \ldots, w_{r 1}, \ldots, w_{r m_{r}}, z_{1}, \ldots, z_{n}\right) .
$$

注 对于上述 Bergman-Hartogs 型域 $\Omega$, 在 $r \geqslant 1$ 为正整数, $p_{1}, \ldots, p_{r}>1, q>0$ 为实数的条件 下, 容易证明 $\Omega$ 不全纯等价于单位球.

下面我们给出几个变换群. 在第 2 节, 我们将证明它们为 (1.1) 式所示的 Bergman-Hartogs 型域 $\Omega$ 的全纯自同构子群.

设

$$
G_{1}=\left\{F: F(w, z)=\left(w_{(1)} \operatorname{det} J_{\tau}(z)^{\frac{q}{p_{1}}}, \ldots, w_{(r)} \operatorname{det} J_{\tau}(z)^{\frac{q}{p_{r}}}, \tau(z)\right), \tau \in \operatorname{Aut}(D),(w, z) \in \Omega\right\},
$$

其中 $\operatorname{det} J_{\tau}$ 是 $D$ 上全纯自同构变换 $\tau(z)$ 的 Jacobi 行列式.

设

$$
G_{2}=\left\{F: F(w, z)=(w, z)\left(\begin{array}{cc}
U & 0 \\
0 & I_{n}
\end{array}\right),(w, z) \in \Omega\right\}
$$

其中

$$
U=\left(\begin{array}{ccc}
U_{m_{1}} & & 0 \\
& \ddots & \\
& & \\
0 & & U_{m_{r}}
\end{array}\right),
$$

$U_{m_{1}}, \ldots, U_{m_{r}}$ 分别是 $m_{1}, \ldots, m_{r}$ 阶西方阵, $I_{n}$ 是 $n$ 阶单位方阵.

设 $E_{i, j}$ 是第 $i$ 行第 $j$ 列元素为 1 其他元素都是 0 的 $m$ 阶方阵, $1 \leqslant i, j \leqslant m$, 则 $m$ 阶初等矩阵 $P_{i, j}$ 为

$$
P_{i, j}=I_{m}-E_{i, i}-E_{j, j}+E_{i, j}+E_{j, i} .
$$

即 $P_{i, j}$ 是由 $m$ 阶单位方阵的第 $i$ 列和第 $j$ 列互换得到的初等矩阵.

如果 $\Omega$ 中点的变量 $w_{(1)}, \ldots, w_{(r)}$ 中有两组变量 $w_{(j)}$ 和 $w_{(k)}$ 的维数和幂次相同, 即 $m_{j}=m_{k}, p_{j}=$ $p_{k}, 1 \leqslant j<k \leqslant r$, 那么定义一个初等变换矩阵

$$
Q_{j, k, l} \triangleq P_{\alpha+1, \beta+1} P_{\alpha+2, \beta+2} \cdots P_{\alpha+l, \beta+l},
$$

其中 $\alpha=m_{1}+\cdots+m_{j-1}, \beta=m_{1}+\cdots+m_{k-1}, l=m_{j}$. 即 $Q_{j, k, l}$ 是将单位方阵 $I_{m}$ 的第 $\alpha+1, \ldots, \alpha+m_{j}$ 列与 $\beta+1, \ldots, \beta+m_{k}$ 列依次对应交换位置, 也就是变量 $w_{(1)}, \ldots, w_{(j)}, \ldots, w_{(k)}, \ldots, w_{(r)}$ 在 $Q_{j, k, l}$ 的 作用下交换 $w_{(j)}$ 和 $w_{(k)}$ 的位置.

记由满足条件 $m_{j}=m_{k}, p_{j}=p_{k}, 1 \leqslant j<k \leqslant r$ 的 $Q_{j, k, l}$ 按照矩阵的乘法运算生成的循环群为 $S$, 即

$$
S=\left\langle Q_{j, k, l}: m_{j}=m_{k}, p_{j}=p_{k}, 1 \leqslant j<k \leqslant r\right\rangle,
$$


设

$$
G_{3}=\left\{F: F(w, z)=(w, z)\left(\begin{array}{cc}
Q & 0 \\
0 & I_{n}
\end{array}\right), Q \in S,(w, z) \in \Omega\right\} .
$$

现在我们给出本文的主要结论:

定理 1 设 $\Omega$ 是 $\mathbb{C}^{m+n}$ 中如 (1.1) 所示的 Bergman-Hartogs 型域. 当 $r=1$, 则 $\Omega$ 的最大全纯自 同构群为 $G_{1} G_{2} G_{3}$, 即 $\operatorname{Aut}(\Omega)=G_{1} G_{2} G_{3}$, 其中, $G_{1}, G_{2}$ 和 $G_{3}$ 分别为 (1.2), (1.3) 和 (1.4) 所给出的变 换群.

定理 2 设 $\Omega$ 是 $\mathbb{C}^{m+n}$ 中如 (1.1) 所示的 Bergman-Hartogs 型域. 若

$$
F \in \operatorname{Aut}(\Omega):(0, z) \rightarrow\left(0, z^{*}\right)
$$

其中 $z, z^{*} \in D$, 则 $F \in G_{1} G_{2} G_{3}$.

\section{2 基础知识}

下面先给出齐性域和对称域的概念.

定义 1 设 $\mathcal{D}$ 是 $\mathbb{C}^{N}$ 中的域. 若对域 $\mathcal{D}$ 中的任意两点 $a$ 和 $b$, 总存在 $\mathcal{D}$ 的一个全纯自同构变换 $F$, 使得 $F(a)=b$. 则称 $\mathcal{D}$ 为齐性域.

定义 2 设 $\mathcal{D}$ 是 $\mathbb{C}^{N}$ 中的一个域. 若对域 $\mathcal{D}$ 内的每一点 $a_{0}$, 都存在域 $\mathcal{D}$ 的全纯自同构变换 $F$, 使得此变换连续作用两次为恒等变换, 且 $a_{0}$ 为变换 $F$ 的唯一不动点. 即

$$
F^{2}=I d, \quad F\left(a_{0}\right)=a_{0},
$$

则称 $\mathcal{D}$ 为对称域.

任一有界对称域必然是齐性域. 不可约的有界对称域又分为四类 Cartan 域和 16 维与 27 维的两 个例外 Cartan 域.

定义 3 设 $\mathcal{D}$ 是 $\mathbb{C}^{N}$ 中的域. 若对于任意 $a \in \mathcal{D}$ 以及实数 $\theta$, 都有 $\mathrm{e}^{\sqrt{-1} \theta} a \in \mathcal{D}$, 则称 $\mathcal{D}$ 为圆型 域.

有界对称域都是圆型域. 根据文献 [7] 可知, (1.1) 式给出的 Bergman-Hartogs 域 $\Omega$ 是分组圆型域, 因此 $\Omega$ 也是圆型域. 对于圆型域上的双全纯映射, 有下面的 Cartan 定理:

Cartan 定理 设 $\mathcal{D}_{1}$ 和 $\mathcal{D}_{2}$ 都是 $\mathbb{C}^{N}$ 中包含原点的圆型域, 并且 $\mathcal{D}_{1}$ 是有界的. 若 $F: \mathcal{D}_{1} \rightarrow \mathcal{D}_{2}$ 是双全纯映射, 且 $F(0)=0$, 则 $F$ 是线性映射.

在 $\mathcal{D}_{1}=\mathcal{D}_{2}$ 时, Cartan 定理即: 对于包含原点的有界圆型域 $\mathcal{D}$, 将原点映为原点的全纯自同构 变换 $F \in \operatorname{Aut}(\mathcal{D})$ 一定是线性映射.

定义 4 设 $\mathcal{D}$ 是 $\mathbb{C}^{N}$ 中具有 $\mathcal{C}^{2}$ 边界的域, $T_{p}^{\mathbb{C}}(b \mathcal{D})$ 是 $\mathcal{D}$ 的边界 $b \mathcal{D}$ 在 $p \in b \mathcal{D}$ 点的复切空间. 如果对所有的复切向量 $\omega \in T_{p}^{\mathbb{C}}(b \mathcal{D})$, 且 $\omega \neq 0$, 有

$$
L_{p}(\rho, \omega)=\sum_{j, k=1}^{m} \frac{\partial^{2} \rho}{\partial z_{j} \partial \bar{z}_{k}}(p) \omega_{j} \bar{\omega}_{k}>0,
$$

则称 $\mathcal{D}$ 在 $p \in b \mathcal{D}$ 是强 Levi 拟凸的, 称 $L_{p}(\rho, \omega)$ 为 Levi 形式. 
下面我们证明第 1 节引入的 $G_{1}, G_{2}$ 和 $G_{3}$ 都是 Bergman-Hartogs 型域 $\Omega$ 的自同构子群, 并且给 出有关 Bergman-Hartogs 型域 $\Omega$ 的性质.

命题 1 设 $\Omega$ 为如 (1.1) 所示的 Bergman-Hartogs 型域, $G_{1}$ 为 (1.2) 所给的集合. 则 $G_{1}$ 是 $\Omega$ 的 一个全纯自同构子群.

证明 对于 $F \in G_{1}$, 有

$$
F(w, z)=\left(w_{(1)} \operatorname{det} J_{\tau}(z)^{\frac{q}{p_{1}}}, \ldots, w_{(r)} \operatorname{det} J_{\tau}(z)^{\frac{q}{p_{r}}}, \tau(z)\right) \triangleq\left(w_{(1)}^{*}, \ldots, w_{(r)}^{*}, z^{*}\right), \quad(w, z) \in \Omega .
$$

利用 $D$ 上 Bergman 核函数的变换法则

$$
K_{D}(z, z)=K_{D}(\tau(z), \tau(z))\left|\operatorname{det} J_{\tau}(z)\right|^{2}, \quad z \in D,
$$

则有

$$
\begin{aligned}
& \left(\left\|w_{(1)}^{*}\right\|^{2 p_{1}}+\cdots+\left\|w_{(r)}^{*}\right\|^{2 p_{r}}\right) K_{D}\left(z^{*}, z^{*}\right)^{q} \\
& \quad=\left(\left\|w_{(1)} \operatorname{det} J_{\tau}(z)^{\frac{q}{p_{1}}}\right\|^{2 p_{1}}+\cdots+\left\|w_{(r)} \operatorname{det} J_{\tau}(z)^{\frac{q}{p_{r}}}\right\|^{2 p_{r}}\right) K_{D}(z, z)^{q}\left|\operatorname{det} J_{\tau}(z)\right|^{-2 q} \\
& \quad=\left(\left\|w_{(1)}\right\|^{2 p_{1}}+\cdots+\left\|w_{(r)}\right\|^{2 p_{r}}\right) K_{D}(z, z)^{q}<1 .
\end{aligned}
$$

因此 $F(w, z)=\left(w_{(1)}^{*}, \ldots, w_{(r)}^{*}, z^{*}\right) \in \Omega$. 显然, $F$ 是全纯的.

令 $F^{-1}(w, z)=\left(w_{(1)} \operatorname{det} J_{\tau^{-1}}(z)^{\frac{q}{p_{1}}}, \ldots, w_{(r)} \operatorname{det} J_{\tau^{-1}}(z)^{\frac{q}{p_{r}}}, \tau^{-1}(z)\right)$, 因为 $\tau \in \operatorname{Aut}(D)$ 是 $D$ 上的全 纯自同构变换, 所以存在 $\tau^{-1} \in \operatorname{Aut}(D)$, 于是 $F^{-1} \in G_{1}$. 故 $F \in G_{1}$ 是 $\Omega \rightarrow \Omega$ 的一一全纯映射, 即 $F \in \operatorname{Aut}(\Omega)$ 且 $F^{-1} \in \operatorname{Aut}(\Omega)$.

另外, 定义 $G_{1}$ 的二元运算为两个映射的复合, 且 $G_{1}$ 的单位元为恒等映射. 于是 $G_{1}$ 是 $\Omega$ 的一个 全纯自同构子群.

命题 2 设 $\Omega$ 为 (1.1) 所示的 Bergman-Hartogs 型域, $G_{2}$ 为 (1.3) 所述的集合. 则 $G_{2}$ 是 $\Omega$ 的一 个全纯自同构子群.

证明 根据矩阵乘法的性质, 要想证明 $G_{2}$ 是 $\Omega$ 的一个全纯自同构子群, 只需证明 $F \in G_{2}$ 是 $\Omega \rightarrow \Omega$ 的映射以及它的可逆性. 对于 $F \in G_{2}$, 有

$$
F(w, z)=(w, z)\left(\begin{array}{cc}
U & 0 \\
0 & I_{n}
\end{array}\right), \quad(w, z) \in \Omega .
$$

根据 $U$ 的定义有

$$
w_{(1)}^{*}=w_{(1)} U_{m_{1}}, \quad \ldots, \quad w_{(r)}^{*}=w_{(r)} U_{m_{r}}, \quad z^{*}=z I_{n},
$$

于是可得

$$
\begin{aligned}
& \left(\left\|w_{(1)}^{*}\right\|^{2 p_{1}}+\cdots+\left\|w_{(r)}^{*}\right\|^{2 p_{r}}\right) K_{D}\left(z^{*}, z^{*}\right) \\
& \quad=\left(\left\|w_{(1)} U_{m_{1}}\right\|^{2 p_{1}}+\cdots+\left\|w_{(r)} U_{m_{r}}\right\|^{2 p_{r}}\right) K_{D}\left(z^{*}, z^{*}\right) \\
& \quad=\left(\left\|w_{(1)}\right\|^{2 p_{1}}+\cdots+\left\|w_{(r)}\right\|^{2 p_{r}}\right) K_{D}(z, z)<1 .
\end{aligned}
$$

因此 $F(w, z)=\left(w^{*}, z^{*}\right) \in \Omega$. 而变换 $F$ 的全纯性是显然的. 现定义

$$
F^{-1}(w, z)=(w, z)\left(\begin{array}{cc}
\bar{U}^{t} & 0 \\
0 & I_{n}
\end{array}\right), \quad(w, z) \in \Omega .
$$


即得 $F^{-1} \in G_{2}$ 且全纯. 由此可知, $F$ 是 $\Omega \rightarrow \Omega$ 的单满全纯映射, 即 $F \in \operatorname{Aut}(\Omega)$.

命题 3 设 $\Omega$ 为 (1.1) 所示的 Bergman-Hartogs 型域, $G_{3}$ 为 (1.4) 所给的集合. 则 $G_{3}$ 是 $\Omega$ 的一 个全纯自同构子群.

证明 类似于命题 2 , 我们只需证明 $\forall F \in G_{3}$ 是 $\Omega \rightarrow \Omega$ 的映射. 设 $\forall F \in G_{3}$, 则有

$$
F(w, z)=(w, z)\left(\begin{array}{cc}
Q & 0 \\
0 & I_{n}
\end{array}\right)
$$

$Q \in S$. 由 $S$ 定义可知, 只需验证 $S$ 的生成元的性质即可. 故我们验证 $Q=Q_{j, k, l}$ 时的情况.

设

$$
F(w, z)=(w, z)\left(\begin{array}{cc}
Q_{j, k, l} & 0 \\
0 & I_{n}
\end{array}\right)=\left(w^{*}, z^{*}\right)
$$

此时, $m_{j}=m_{k}, p_{j}=p_{k}$. 则

$$
\begin{aligned}
& \left(w_{(1)}^{*}, \ldots, w_{(j-1)}^{*}, w_{(j)}^{*}, w_{(j+1)}^{*}, \ldots, w_{(k-1)}^{*}, w_{(k)}^{*}, w_{(k+1)}^{*}, \ldots, w_{(r)}^{*}, z^{*}\right) \\
& \quad=\left(w_{(1)}, \ldots, w_{(j-1)}, w_{(j)}, w_{(j+1)}, \ldots, w_{(k-1)}, w_{(k)}, w_{(k+1)}, \ldots, w_{(r)}, z\right)\left(\begin{array}{cc}
Q_{j, k, l} & 0 \\
0 & I_{n}
\end{array}\right) \\
& \quad=\left(w_{(1)}, \ldots, w_{(j-1)}, w_{(k)}, w_{(j+1)}, \ldots, w_{(k-1)}, w_{(j)}, w_{(k+1)}, \ldots, w_{(r)}, z\right) .
\end{aligned}
$$

由此可得

$$
\begin{aligned}
& \left(\left\|w_{(1)}^{*}\right\|^{2 p_{1}}+\cdots+\left\|w_{(r)}^{*}\right\|^{2 p_{r}}\right) K_{D}\left(z^{*}, z^{*}\right) \\
& \quad=\left(\left\|w_{(1)}\right\|^{2 p_{1}}+\cdots+\left\|w_{(r)}\right\|^{2 p_{r}}\right) K_{D}(z, z)<1 .
\end{aligned}
$$

此即 $\left(w^{*}, z^{*}\right) \in \Omega$. 另外, $F^{-1}=F$, 所以 $G_{3}$ 是 $\operatorname{Aut}(\Omega)$ 的一个子群.

命题 4 设 $\Omega$ 为 (1.1) 所示的 Bergman-Hartogs 型域. 若 $F \in \operatorname{Aut}(\Omega)$ 且满足 $F(0,0)=(0,0)$ 和 $F(w, z)=\left(w^{*}, z\right),(w, z) \in \Omega$, 则 $F \in G_{2} G_{3}$.

证明 我们需要先引入 $\mathbb{C}^{r}$ 中的 Egg 域

$$
\Omega^{*}=\left\{\left(w_{1}, \ldots, w_{r}\right) \in \mathbb{C}^{r}:\left|w_{1}\right|^{2 p_{1}}+\cdots+\left|w_{r}\right|^{2 p_{r}}<c\right\},
$$

其中 $c>0$ 是常数, $r \geqslant 1$ 为正整数, 参数 $p_{1}, \ldots, p_{r}>1$ 为实数. 由 Liu 和 Tang 的结果可知: $\Omega^{*}$ 在原 点的迷向子群为

$$
G^{*}=\left\{f: f\left(w_{1}, \ldots, w_{r}\right)=\left(w_{1}, \ldots, w_{r}\right) U^{*} Q^{*}\right\}
$$

其中

$$
U^{*}=\left(\begin{array}{ccc}
\mathrm{e}^{\sqrt{-1} \theta_{1}} & & 0 \\
& \ddots & \\
0 & & \mathrm{e}^{\sqrt{-1} \theta_{r}}
\end{array}\right),
$$

$\theta_{j} \in \mathbb{R}, j=1, \ldots, r$ 和 $Q^{*} \in\left\langle P_{i, j}^{*}: p_{i}=p_{j}\right\rangle, P_{i, j}^{*}$ 是由 $r$ 阶单位方阵的第 $i$ 列和第 $j$ 列互换得到的初 等矩阵.

我们再引入 $\mathbb{C}^{m}$ 中的 $\operatorname{Egg}$ 域 $\widehat{\Omega}$,

$$
\widehat{\Omega}=\left\{w=\left(w_{(1)}, \ldots, w_{(r)}\right) \in \mathbb{C}^{m_{1}} \times \cdots \times \mathbb{C}^{m_{r}}:\left\|w_{(1)}\right\|^{2 p_{1}}+\cdots+\left\|w_{(r)}\right\|^{2 p_{r}}<c\right\},
$$


其中 $c>0$ 为常数, $r \geqslant 1$ 为正整数, 参数 $p_{1}, \ldots, p_{r}>1$ 为实数, 且 $w_{(j)}=\left(w_{j 1}, \ldots, w_{j m_{j}}\right) \in \mathbb{C}^{m_{j}}, j=$ $1, \ldots, r$. 对于 $m_{j}$ 阶酉方阵 $U_{m_{j}}$, 有

$$
\left\|w_{(j)} U_{m_{j}}\right\|=\left\|w_{(j)}\right\|
$$

对于 $m_{j}=m_{k}, p_{j}=p_{k}$, 有

$$
\begin{gathered}
\left(w_{(1)}, \ldots, w_{(j-1)}, w_{(j)}, w_{(j+1)}, \ldots, w_{(k-1)}, w_{(k)}, w_{(k+1)}, \ldots, w_{(r)}, z\right)\left(\begin{array}{cc}
Q_{j, k, l} & 0 \\
0 & I_{n}
\end{array}\right) \\
=\left(w_{(1)}, \ldots, w_{(j-1)}, w_{(k)}, w_{(j+1)}, \ldots, w_{(k-1)}, w_{(j)}, w_{(k+1)}, \ldots, w_{(r)}, z\right) .
\end{gathered}
$$

因此根据 $\Omega^{*}$ 的原点处的迷向子群 $G^{*}$ 以及 $U$ 和 $Q$ 的定义, 易得 $\widehat{\Omega}$ 在原点处的迷向子群为

$$
\widehat{G}=\left\{f: f\left(w_{1}, \ldots, w_{r}\right)=\left(w_{1}, \ldots, w_{r}\right) U Q\right\},
$$

其中

$$
U=\left(\begin{array}{ccc}
U_{m_{1}} & & 0 \\
& \ddots & \\
& & \\
0 & & U_{m_{r}}
\end{array}\right)
$$

且 $Q \in S=\left\langle Q_{j, k, l}: m_{j}=m_{k}, p_{j}=p_{k}, 1 \leqslant j<k \leqslant r\right\rangle$.

对于 $\mathbb{C}^{m+n}$ 中的 Bergman-Hartogs 型域 $\Omega$, 如果 $\Omega$ 中的点 $(w, z)$ 的分量 $z=0$, 那么得到的 $\Omega$ 的 子集与 $\widehat{\Omega}$ 是相同的. 从而易得命题结论.

为了研究如 (1.1) 所示的 Bergman-Hartogs 域 $\Omega$ 的边界的拟凸性, 我们将 $\Omega$ 的边界分成不相交 的两部分. 设 $\Omega$ 的边界为 $b \Omega$ 并记 $\rho(w, z)=\left\|w_{(1)}\right\|^{2 p_{1}}+\cdots+\left\|w_{(r)}\right\|^{2 p_{r}}-K_{D}(z, z)^{-q}$, 则

$$
\begin{aligned}
b \Omega= & \left\{(w, z) \in \mathbb{C}^{m_{1}} \times \cdots \times \mathbb{C}^{m_{r}} \times \bar{D}: \rho(w, z)=0, z \in \bar{D}\right\} \\
= & \left\{(w, z) \in \mathbb{C}^{m_{1}} \times \cdots \times \mathbb{C}^{m_{r}} \times \bar{D}: \rho(w, z)=0, w=0, z \in \bar{D}\right\} \\
& \cup\left\{(w, z) \in \mathbb{C}^{m_{1}} \times \cdots \times \mathbb{C}^{m_{r}} \times \bar{D}: \rho(w, z)=0, w \neq 0, z \in \bar{D}\right\} \\
= & \left\{(w, z): w \in \mathbb{C}^{m_{1}} \times \cdots \times \mathbb{C}^{m_{r}}, w=0, z \in b D\right\} \\
& \cup\left\{(w, z): w \in \mathbb{C}^{m_{1}} \times \cdots \times \mathbb{C}^{m_{r}}, \rho(w, z)=0, w \neq 0, z \in D\right\},
\end{aligned}
$$

其中, $\bar{D}$ 表示 $D$ 的闭包, $b D$ 表示 $D$ 的边界. 分别记

$$
\begin{aligned}
& b_{0} \Omega=\left\{(w, z): 0=w \in \mathbb{C}^{m_{1}} \times \cdots \times \mathbb{C}^{m_{r}}, z \in b D\right\}=\{0\} \times b D, \\
& b_{1} \Omega=\left\{(w, z) \in \mathbb{C}^{m_{1}} \times \cdots \times \mathbb{C}^{m_{r}} \times D: \rho(w, z)=0, w \neq 0\right\} .
\end{aligned}
$$

即得

$$
b \Omega=b_{0} \Omega \cup b_{1} \Omega .
$$

命题 5 设 $\Omega$ 为 (1.1) 式所示的 Bergman-Hartogs 型域, 若 $r=1$, 则 $b_{1} \Omega$ 上的点都是强拟凸边 界点.

证明 令 $\rho(w, z)=\left\|w_{(1)}\right\|^{2 p_{1}}-K_{D}(z, z)^{-q}$. 对于 $\left(w_{0}, z_{0}\right) \in b_{1} \Omega$, 有 $z_{0} \in D$ 且 $w_{0}=w_{(1) 0} \neq 0$. 记 $V\left(z_{0}\right) \in D$ 是 $z_{0}$ 在 $D$ 上的邻域, 且 $\triangle\left(w_{(1) 0}\right)$ 是 $w_{(1) 0}$ 在 $\mathbb{C}^{m_{1}}$ 中的邻域. 则 $\left(w_{0}, z_{0}\right)$ 点的邻域 $U\left(w_{0}, z_{0}\right)=V\left(z_{0}\right) \times \triangle\left(w_{(1) 0}\right)$ 满足 
(1) $U\left(w_{0}, z_{0}\right) \cap \Omega=\left\{(w, z) \in U\left(w_{0}, z_{0}\right): \rho(w, z)<0\right\}$;

(2) 对于 $\forall(w, z) \in U\left(w_{0}, z_{0}\right)$, 有 $d \rho(w, z) \neq 0$.

由此可知 $\rho(w, z)$ 是 $\Omega$ 在 $\left(w_{0}, z_{0}\right)$ 点的局部定义函数. 要证明 $\left(w_{0}, z_{0}\right) \in b_{1} \Omega$ 点为强拟凸边界点, 需要我们计算 $\rho$ 在 $\left(w_{0}, z_{0}\right)$ 点处的 Levi 形式.

对于 $\forall\left(w_{0}, z_{0}\right) \in b_{1} \Omega$, 有 $z_{0} \in D$, 则存在有界对称域 $D$ 上的全纯自同构变换 $\tau_{z_{0}} \in \operatorname{Aut}(D)$, 使得 $\tau_{z_{0}}(z)=z^{*}$ 和 $\tau_{z_{0}}\left(z_{0}\right)=0$. 于是, 由 $G_{1}$ 的定义 (1.2) 式可知, 存在 $\Omega$ 上的可以延拓到边界的全纯自同 构变换

$$
F \in G_{1}: F(w, z)=\left(w^{*}, z^{*}\right),
$$

其中 $z^{*}=\tau_{z_{0}}(z)$, 使得 $F\left(w_{0}, z_{0}\right)=\left(w_{0}^{*}, 0\right) \in b_{1} \Omega$. 再根据 $\rho(w, z)$ 的 Hessian 矩阵 $H(w, z)$ 在映射 $F \in G_{1}$ 下的变换公式可得

$$
H\left(w_{0}, z_{0}\right)=\left.\left.J_{F}(w, z)\right|_{\left(w_{0}, z_{0}\right)} H\left(w_{0}^{*}, 0\right) \overline{J_{F}(w, z)}\right|_{\left(w_{0}, z_{0}\right)},
$$

因此若要验证 $\rho$ 在 $\left(w_{0}, z_{0}\right) \in b_{1} \Omega$ 处的 Levi 形式, 只需验证 $\rho$ 在 $\left(w_{0}^{*}, 0\right) \in b_{1} \Omega$ 点的 Levi 形式.

注意到,

$$
\frac{\partial^{2} \rho}{\partial w_{1 k} \partial \bar{w}_{1 k}}=p_{1}\left(p_{1}-1\right)\left\|w_{(1)}\right\|^{2\left(p_{1}-2\right)}\left|w_{1 k}\right|^{2}+p_{1}\left\|w_{(1)}\right\|^{2\left(p_{1}-1\right)},
$$

其中 $k=1, \ldots, m_{1}$.

$$
\frac{\partial^{2} \rho}{\partial w_{1 k} \partial \bar{w}_{1 l}}=p_{1}\left(p_{1}-1\right)\left\|w_{(1)}\right\|^{2\left(p_{1}-2\right)} w_{1 k} \bar{w}_{1 l},
$$

其中 $k, l=1, \ldots, m_{1}$. 有界对称域 $D$ 的 Bergman 核函数 $K_{D}(z, z)$ 满足 $K(z, 0)=K(0,0)$. 因而有

$$
\left.\frac{\partial K_{D}(z, z)}{\partial z}\right|_{z=0}=\left.\frac{\partial K_{D}(z, 0)}{\partial z}\right|_{z=0}=0 .
$$

又因为 $D$ 上的 Bergman 度量矩阵为

$$
T(z)=\left(\frac{\partial^{2} \log K_{D}(z, z)}{\partial z_{j} \partial \bar{z}_{k}}\right)_{1 \leqslant j, k \leqslant n}, \quad z \in D,
$$

所以综合以上内容, 我们得 $\rho$ 在 $\left(w_{0}^{*}, 0\right)$ 处的 Hessian 矩阵 $H\left(w_{0}^{*}, 0\right)$ 为

$$
H\left(w_{0}^{*}, 0\right)=\left(\begin{array}{cc}
\left.M\right|_{w=w_{0}^{*}} & 0 \\
0 & c_{1} T(0)
\end{array}\right)
$$

其中 $M=p_{1}\left\|w_{(1)}\right\|^{2\left(p_{1}-1\right)}\left(\frac{\left(p_{1}-1\right)}{\left\|w_{(1)}\right\|^{2}} w_{(1)}^{t} \bar{w}_{(1)}+I_{m_{1}}\right)$ 和 $c_{1}=\frac{q}{K_{D}(0,0)^{q}}$. 由于

$$
\begin{aligned}
\left.\operatorname{det} M\right|_{w=w_{0}^{*}} & =\left(p_{1}\left\|w_{(1) 0}^{*}\right\|^{2\left(p_{1}-1\right)}\right)^{m_{1}}\left(1+\frac{\left(p_{1}-1\right)}{\left\|w_{(1) 0}^{*}\right\|^{2}}\left\|w_{(1) 0}^{*}\right\|^{2}\right) \\
& =p_{1}^{m_{1}+1}\left\|w_{(1) 0}^{*}\right\|^{2\left(p_{1}-1\right) m_{1}}>0,
\end{aligned}
$$

$q>0, T(0)>0$ 和 $K_{D}(0,0)>0$, 显然, $H\left(w_{0}^{*}, 0\right)>0$.

由 $H\left(w_{0}^{*}, 0\right)>0$ 立即可得

$$
H\left(w_{0}, z_{0}\right)>0, \quad\left(w_{0}, z_{0}\right) \in b_{1} \Omega .
$$

证明完毕. 
现在我们给出定理证明中要用到的一个引理.

引理 1 设 $\Omega$ 为 (1.1) 所示的 Bergman-Hartogs 型域, 记 $\Omega$ 的子集 $\{(w, z) \in \Omega: w=0\}:=\{0\} \times D$. 当 $r=1$ 时, 若 $F \in \operatorname{Aut}(\Omega)$, 则 $F$ 将 $\{0\} \times D$ 映射到 $\{0\} \times D$, 即对于 $\forall(0, z) \in\{0\} \times D \subset \Omega$, 有 $F(0, z)=\left(0, z^{*}\right) \in\{0\} \times D$.

证明 设 $F \in \operatorname{Aut}(\Omega)$, 令 $\left\{\left(0, z^{j}\right)\right\}_{j=1}^{\infty}$ 是 $\{0\} \times D$ 上任意一列收玫到边界 $b_{0} \Omega=\{0\} \times b D$ 上点 的点列, 其中 $z^{j} \in D$. 则我们断定 $\left\{F\left(0, z^{j}\right)\right\}_{j=1}^{\infty}$ 的收玫子列也收玫到边界 $b_{0} \Omega=\{0\} \times b D$ 上的点.

事实上, 由于 $D$ 是有界对称域, 因而存在 $\tau \in \operatorname{Aut}(D)$, 使得 $\tau\left(z^{j}\right)=0$, 于是由命题 1 可知, 存在 $\Psi_{j} \in G_{1}$, 使得

$$
\Psi_{j}(0,0)=\left(0, z^{j}\right), \quad j=1, \ldots, \infty .
$$

故 $\left\{F\left(0, z^{j}\right)\right\}_{j=1}^{\infty}$ 可以被看作是一个全纯自同构轨道 $\left\{\left(F \circ \Psi_{j}\right)(0,0)\right\}_{j=1}^{\infty}$. 如果 $\left\{F\left(0, z^{j}\right)\right\}_{j=1}^{\infty}$ 有一收敛 到边界 $b_{1} \Omega$ 的子列, 不妨设这个子列收玫到点 $p \in b_{1} \Omega$, 那么由命题 5 可知, 点 $p$ 是强拟凸点. 应用 Wong-Rosay 定理 ${ }^{[8,9]}$ : 对于 $\mathbb{C}^{N}$ 中的有界域 $\mathcal{D}, p$ 是 $\Omega$ 的强拟凸边界点. 若存在一列全纯自同构变 换 $F_{k} \in \operatorname{Aut}(\Omega)$ 和 $\Omega$ 的内点 $a$, 使得

$$
\lim _{k \rightarrow \infty} F_{k}(a)=p
$$

则 $\mathcal{D}$ 全纯等价于 $\mathbb{C}^{N}$ 中的单位球 $B_{N}$. 由此可知, Bergman-Hartogs 域 $\Omega$ 一定全纯等价于 $\mathbb{C}^{m+n}$ 中的单位球 $B_{n}$. 而这与 $\Omega$ 的定义 (1.1) 矛盾. 因此, $\left\{F\left(0, z^{j}\right)\right\}_{j=1}^{\infty}$ 的收玫子列也一定收敛到边界 $b_{0} \Omega=\{0\} \times b D$ 上的点. 故对于 $\Omega$ 上所有收玫到边界 $b_{0} \Omega$ 的点列 $\left\{\left(0, z^{j}\right)\right\}_{j=1}^{\infty}$, 有 $\left\{F\left(0, z^{j}\right)\right\}_{j=1}^{\infty}$ 的收 敛子列也收敛到边界 $b_{0} \Omega$ 上.

设 $F \in \operatorname{Aut}(\Omega):(w, z) \rightarrow\left(w^{*}, z^{*}\right)$. 记 $F(w, z)=\left(f_{11}(w, z), \ldots, f_{1 m_{1}}(w, z), g_{1}(w, z), \ldots, g_{n}(w, z)\right)$. 则

$$
F(0, z)=\left(f_{11}(0, z), \ldots, f_{1 m_{1}}(0, z), g_{1}(0, z), \ldots, g_{n}(0, z)\right), \quad(0, z) \in\{0\} \times D
$$

定义

$$
h: D \rightarrow \mathbb{C}, \quad h(z)=f_{11}(0, z), \quad z \in D .
$$

对于 $\forall z^{*} \in b D$, 有 $\lim _{j \rightarrow \infty} z^{j}=z^{*}$. 于是根据前面我们的断言可知, 对于收玫子列 $\left\{F\left(0, z^{j k}\right)\right\}_{j, k=1}^{\infty} \subset$ $\left\{F\left(0, z^{j}\right)\right\}_{j=1}^{\infty} \subset D$, 有

$$
\lim _{k \rightarrow \infty} h\left(z^{j k}\right)=\lim _{k \rightarrow \infty} f_{11}\left(0, z^{j k}\right)=0 .
$$

故 $h$ 可以连续延拓到 $D$ 的边界 $b D$. 再由 $z^{*}$ 任意性可知,

$$
h\left(z^{*}\right)=0, \quad \forall z^{*} \in b D .
$$

从而由最大模原理可知 $h(z)=f_{11}(0, z) \equiv 0, z \in D$. 同理可得, $f_{12}(0, z)=\cdots=f_{1 m_{1}}(0, z) \equiv 0, z \in$ $D$. 此即

$$
F(0, z)=(0, g(0, z))=\left(0, z^{*}\right) \in\{0\} \times D, \quad(0, z) \in\{0\} \times D .
$$




\section{3 定理证明}

现在我们要证明本文的主要定理.

证明 由命题 1-3 即知

$$
G_{1} G_{2} G_{3} \subseteq \operatorname{Aut}(\Omega) .
$$

因而, 我们只需证 $\operatorname{Aut}(\Omega) \subseteq G_{1} G_{2} G_{3}$.

首先, 对于 $F \in \operatorname{Aut}(\Omega)$ 为 Bergman-Hartogs 型域 $\Omega$ 的任意一个全纯变换, 对于定理 1 , 由引理 1 可知

$$
F(0,0)=\left(0, z_{0}\right), \quad\left(0, z_{0}\right) \in\{0\} \times D .
$$

对于定理 2, 由条件 (1.5) 也可得 (3.1) 式. 选取映射

$$
\Psi \in G_{1}: \Psi(w, z)=\left(w_{(1)} \operatorname{det} J_{\tau_{z_{0}}}(z)^{\frac{q}{p_{1}}}, \ldots, w_{(r)} \operatorname{det} J_{\tau_{z_{0}}}(z)^{\frac{q}{p_{r}}}, \tau_{z_{0}}(z)\right), \quad(w, z) \in \Omega,
$$

其中 $\tau_{z_{0}}\left(z_{0}\right)=0$, 使得

$$
\Psi\left(0, z_{0}\right)=(0,0) .
$$

因此,

$$
(\Psi \circ F) \in \operatorname{Aut}(\Omega)
$$

且满足

$$
(\Psi \circ F)(0,0)=(0,0) .
$$

其次, 根据以上证明, 对于 $\forall F \in \operatorname{Aut}(\Omega)$, 不妨设 $F(0,0)=(0,0)$, 记

$$
F(w, z)=\left(w^{*}, z^{*}\right), \quad(w, z) \in \Omega,
$$

其中

$$
w^{*}=f(w, z)=\left(f_{(1)}(w, z), \ldots, f_{(r)}(w, z)\right), \quad z^{*}=g(w, z) .
$$

下面我们证明 $g(0, z) \in \operatorname{Aut}(D)$.

对于定理 1 , 应用引理 1 可知

$$
F(0, z)=\left(f_{(1)}(0, z), \ldots, f_{(r)}(0, z), g(0, z)\right)=\left(0, z^{*}\right), \quad(0, z) \in\{0\} \times D
$$

(对于定理 2, 由条件 (1.5) 可得 (3.4) 式), 即

$$
w^{*}=\left(f_{(1)}(0, z), \ldots, f_{(r)}(0, z)\right)=0, \quad z^{*}=g(0, z) .
$$

由此可知 $g(0, z)$ 是由 $D$ 到 $D$ 的映射. 对于任意的 $z \in D, \widetilde{z} \in D$, 且 $z \neq \widetilde{z}$, 则有 $(0, z),(0, \widetilde{z}) \in \Omega$, 且 $(0, z) \neq(0, \widetilde{z})$. 而 $F$ 是 $\Omega$ 到 $\Omega$ 的全纯一一映射, 故 $F(0, z) \neq F(0, \widetilde{z})$, 即 $\left(0, z^{*}\right) \neq\left(0, \widetilde{z}^{*}\right)$. 从而

$$
g(0, z)=z^{*} \neq \widetilde{z}^{*}=g(0, \widetilde{z}) .
$$

因此 $g(0, z)$ 是单射. 对于 $\forall z^{*} \in D$, 有 $\left(0, z^{*}\right) \in \Omega$, 则存在 $(0, z) \in \Omega$, 使得

$$
F^{-1}\left(0, z^{*}\right)=(0, z)
$$


即存在 $(0, z) \in \Omega$, 使得

$$
F(0, z)=\left(0, z^{*}\right)
$$

从而存在 $z \in D$, 使得

$$
g(0, z)=z^{*} .
$$

因此 $g(0, z)$ 是满射. 显然 $g(0, z)$ 是全纯的. 于是我们得到 $g(0, z) \in \operatorname{Aut}(D)$.

所以存在 $\tau_{0} \in \operatorname{Aut}(D)$, 有

$$
g(0, z)=\tau_{0}(z), \quad \text { 且 } \tau_{0}(0)=0 .
$$

在 $G_{1}$ 中选取映射

$$
\Psi_{1} \in G_{1}: \Psi_{1}(w, z)=\left(w_{(1)} \operatorname{det} J_{\tau_{0}}(z)^{\frac{q}{p_{1}}}, \ldots, w_{(r)} \operatorname{det} J_{\tau_{0}}(z)^{\frac{q}{p_{r}}}, \tau_{0}(z)\right), \quad(w, z) \in \Omega .
$$

显然 $\Psi_{1}(0, z)=\left(0, \tau_{0}(z)\right),(0, z) \in \Omega$.

由 $F(0,0)=(0,0)$ 且 $\Psi_{1}(0,0)=(0,0)$, 故

$$
\Psi_{1}^{-1} \circ F(0,0)=(0,0) .
$$

又由于 $\Omega$ 是有界圆型域, 对此应用 Cartan 定理可知, $\Psi_{1}^{-1} \circ F$ 是线性映射. 因此 $\Psi_{1}^{-1} \circ F$ 的形式为

$$
\left(\Psi_{1}^{-1} \circ F\right)(w, z)=(w, z)\left(\begin{array}{ll}
A & B \\
C & D
\end{array}\right),
$$

其中 $A, B, C$ 和 $D$ 分别为分块矩阵. 联合 (3.3) 和 (3.5) 可知 $\Psi_{1}^{-1} \circ F$ 满足

$$
\left(\Psi_{1}^{-1} \circ F\right)(0, z)=(0, z), \quad(0, z) \in \Omega .
$$

将上式代入 (3.6) 可得

$$
C=0, \quad D=I_{n},
$$

$I_{n}$ 为 $n$ 阶单位方阵. 即 $(3.6)$ 化为

$$
\left(\Psi_{1}^{-1} \circ F\right)(w, z)=(w, z)\left(\begin{array}{cc}
A & B \\
0 & I_{n}
\end{array}\right) .
$$

我们易证 (3.3) 中 $F$ 的分量 $f$ 满足

$$
f(w, 0) \in \operatorname{Aut}(\widehat{\Omega}): w \rightarrow w A,
$$

其中 $\widehat{\Omega}$ 为 $(2.1)$ 式所给的 Egg 域. 再由命题 4 易得存在映射

$$
\Psi_{2} \in G_{2}:(w, z) \rightarrow(w, z)\left(\begin{array}{cc}
U & 0 \\
0 & I_{n}
\end{array}\right) \quad \text { 和 } \quad \Psi_{3} \in G_{3}:(w, z) \rightarrow(w, z)\left(\begin{array}{cc}
Q & 0 \\
0 & I_{n}
\end{array}\right),
$$

使得 $A=U Q$, 即

$$
\Psi_{2} \Psi_{3} \in G_{2} G_{3}:(w, z) \rightarrow(w, z)\left(\begin{array}{cc}
A & 0 \\
0 & I_{n}
\end{array}\right)=(w, z)\left(\begin{array}{cc}
U Q & 0 \\
0 & I_{n}
\end{array}\right)
$$

40 
其中

$$
Q \in S, U=\left(\begin{array}{ccc}
U_{m_{1}} & & 0 \\
& \ddots & \\
& & U_{m_{r}}
\end{array}\right),
$$

$U_{m_{1}}, \ldots, U_{m_{r}}$ 分别是 $m_{1}, \ldots, m_{r}$ 阶西方阵. 于是对

$$
\Psi_{3}^{-1}, \quad \Psi_{2}^{-1} \quad \text { 和 } \quad \Psi_{1}^{-1} \circ F
$$

作复合运算可得

$$
\left(\Psi_{3}^{-1} \circ \Psi_{2}^{-1} \circ \Psi_{1}^{-1} \circ F\right)(w, z)=(w, z)\left(\begin{array}{cc}
I_{m} & B \\
0 & I_{n}
\end{array}\right) .
$$

若 $B \neq 0$, 则对映射 $\Psi_{3}^{-1} \circ \Psi_{2}^{-1} \circ \Psi_{1}^{-1} \circ F$ 连续作 $k$ 次复合运算后为

$$
\left(\Psi_{3}^{-1} \circ \Psi_{2}^{-1} \circ \Psi_{1}^{-1} \circ F\right)^{k}(w, z)=(w, z)\left(\begin{array}{cc}
I_{m} & k B \\
0 & I_{n}
\end{array}\right) .
$$

而这与有界域在原点的迷向子群是紧的相矛盾, 所以 $B=0$. 因而 (3.7) 式为

$$
\Psi_{3}^{-1} \circ \Psi_{2}^{-1} \circ \Psi_{1}^{-1} \circ F=\mathrm{Id}
$$

即

$$
F=\Psi_{1} \circ \Psi_{2} \circ \Psi_{3} \in G_{1} G_{2} G_{3} .
$$

最后, 由以上结论我们得到, 对于 $\forall F \in \operatorname{Aut}(\Omega)$, 有 $F \in G_{1} G_{2} G_{3}$, 即 $\operatorname{Aut}(\Omega) \subseteq G_{1} G_{2} G_{3}$.

文献 $[4,5,10]$ 研究的有界域都是 (1.1) 式给出的 Bergman-Hartogs 型域的特殊情况, 应用我们的 结果定理 1 可以得到文献 $[4,5,10]$ 中域的全纯自同构群.

致谢 衷心地感谢审稿人对本文提出宝贵意见和建议.

\section{参考文献}

1 华罗庚. 多复变数函数论中的典型域的调和分析. 北京: 科学出版社, 1958

2 Xu Y C. Automorphism group of exceptional symmetric domains $R_{V}$. Sci China Ser A, 2000, 43: 347-356

3 Xu Y C. Automorphism group of exceptional symmetric domains $R_{V I}$. Sci China Ser A, 2000, 43: 1035-1045

4 Ahn H, Byun J, Park J D. Automorphisms of the Hartogs type domains over classical symmetric domains. Int J Math, 2012, 23: 1250098-1-11

5 殷慰萍, 王安, 赵振刚, 等. 华罗庚域的 Bergman 核函数. 中国科学 A 辑, 2001, 31: 503-516

6 Xu Y C, Chen M R, Ma S Y. Explicit formula of holomorphic automorphism group on complex homogeneous bounded domains. Sci China Ser A, 2006, 49: 1392-1404

7 Wang A, Liu Y L. Zeros of the Bergman kernels on some new Hartogs domains. Chin Quart J Math, 2011, 26: 325-334

8 Rosay J P. Sur une caract é risation de la boule parmi les domaines de $\mathbb{C}^{n}$ par son groupe d' automorphismes. Ann Inst Fourier (Grenoble), 1979, 29: 91-97

9 Wong B. Characterization of the unit ball in $\mathbb{C}^{n}$ by its automorphism group. Invent Math, 1977, 41: 253-257

10 殷慰萍. 第一类超 Cartan 域的 Bergman 核函数. 中国科学 A 辑, 1999, 29: 607-615 


\section{The holomorphic automorphism group of the domains of the Bergman-Hartogs type}

\section{PAN LiShuang \& WANG An}

Abstract We consider the domain $\Omega$ of the Bergman-Hartogs type which bases on any bounded symmetric domain $D$,

$$
\Omega=\left\{\left(w_{(1)}, \ldots, w_{(r)}, z\right) \in \mathbb{C}^{m_{1}} \times \cdots \times \mathbb{C}^{m_{r}} \times D:\left\|w_{(1)}\right\|^{2 p_{1}}+\cdots+\left\|w_{(r)}\right\|^{2 p_{r}}<K_{D}(z, z)^{-q}\right\},
$$

where $K_{D}(z, z)$ denotes the Bergman kernel on $D, r$ is a positive integer, $p_{1}, \ldots, p_{r}>1$ and $q>0$ are real parameters. We give the holomorphic automorphism group of $\Omega$, and prove that the given holomorphic automorphism group is the full holomorphic automorphism group of $\Omega$ for $r=1$. In addition, when $r>1$, if the holomorphic automorphism mapping $F$ on $\Omega$ maps $(0, z) \in\{0\} \times D$ to $\left(0, z^{*}\right) \in\{0\} \times D$, then $F$ belongs to the given holomorphic automorphism group.

Keywords domain of the Bergman-Hartogs type, holomorphic automorphism group, bounded symmetric domain

MSC(2010) 32A07, 32M05

doi: 10.1360/N012014-00005 